\title{
A Robust Basis for Grain Identification in Polycrystalline Thin Film Devices Using Cepstrum Transforms of 4D-STEM Diffraction Pattern
}

Xiyue Zhang ${ }^{1}$, Elliot Padgett ${ }^{2}$, Lijun Zhu ${ }^{1}$, Robert Buhrman ${ }^{1}$ and David Muller ${ }^{1}$

${ }^{1}$ Cornell University, Ithaca, New York, United States, ${ }^{2}$ Cornell University, DC, Washington, United States

Scanning nanobeam electron diffraction (NBED) with fast pixelated detectors is a valuable technique for lattice structure mapping, including grain identification [1]. For polycrystalline thin films, there is difficulty in identifying small grain structures using NBED. A thin film requires a small probe size to have good spatial resolution, which means a large convergence angle to focus the electron beam [2]. This results in broad diffraction spots and thus an increased difficulty in resolving the patterns. Here, we apply an approach called the exit wave power cepstrum (EWPC) that cleanly decouples the tilt, thickness and shape factor information from the lattice structure in thin film diffraction patterns, greatly simplifying the classification of small grain structures at arbitrary orientations [1].

A $14 \mathrm{~nm}$-thick polycrystalline Cobalt-Platinum alloy grown by sputtering for use as a spin-Hall device is studied here. We use 4D-STEM and a high-speed electron microscope pixel array detector (EMPAD) for collecting scanning NBED patterns [3]. Figure 1a is an annular bright field STEM image of a crosssectional sample showing the polycrystalline nature of the film and columnar growth of the grains. As the thickness of the cross-section is much larger than the grain size, we can see from figure $1 \mathrm{~b}$ that it is hard to distinguish single grains from overlapping diffraction patterns.

Figure 2 displays how useful the cepstrum transform is in capturing lattice features from overlapping grains and random grain orientations. The mistilt and thickness variation (Fig. 2b) have little effect in the EWPC (Fig. 2d), and thus the power cepstrum is more capable of discerning similar grain structures (Fig. 2c) than the diffraction pattern (Fig. 2a). Figure 2e and $2 \mathrm{f}$ show grain mappings of the first 30 components from Principal Component Analysis (PCA) of the original NBED patterns (Fig. 2e), and after an EWPC transform of the NBED dataset (Fig. 2f) [1]. While figure 2e only identifies a limited set of grains, figure $2 \mathrm{f}$ gives a much more comprehensive view of the columnar growth of the grains, with nanometer spatial resolution. Therefore, 4D-STEM combined with exit wave power cepstrum and principle component analysis provides a robust method for identifying small grain structures in polycrystalline thin film devices at nanometer spatial resolution. [4]

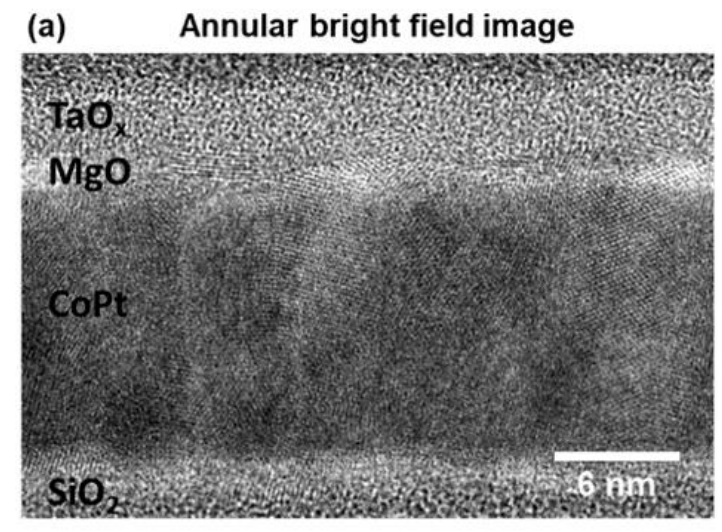

(b) Averaged diffraction pattern

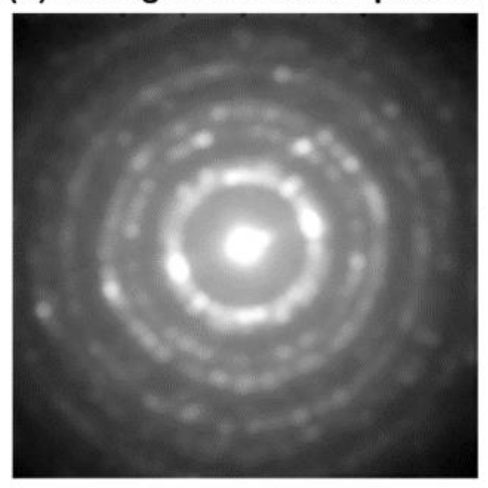


Figure 1. Randomly oriented grain structure in CoPt alloy. (a) An annular bright field STEM image of a cross-section of $\mathrm{SiO} 2 / \mathrm{CoPt} / \mathrm{MgO} / \mathrm{TaOx}$ along the (110) zone axis of the $\mathrm{Si}$ substrate showing the columnar growth of the grains. (b) The averaged diffraction pattern of the CoPt layer from a sum of all 4D-STEM diffraction pattens in the data set, showing the overlapping spots from randomly oriented grains.
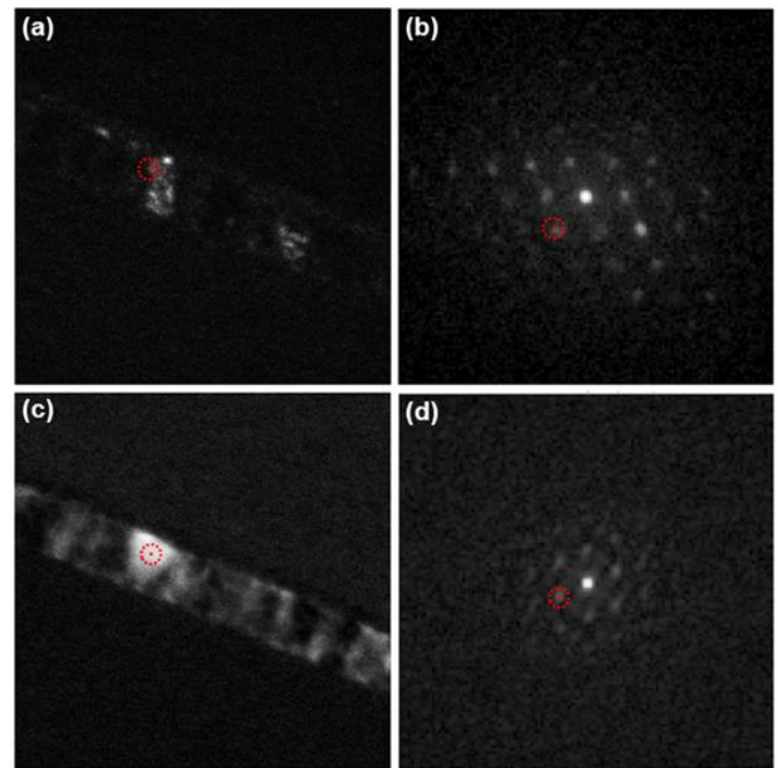

(e) PCA from diffraction pattern
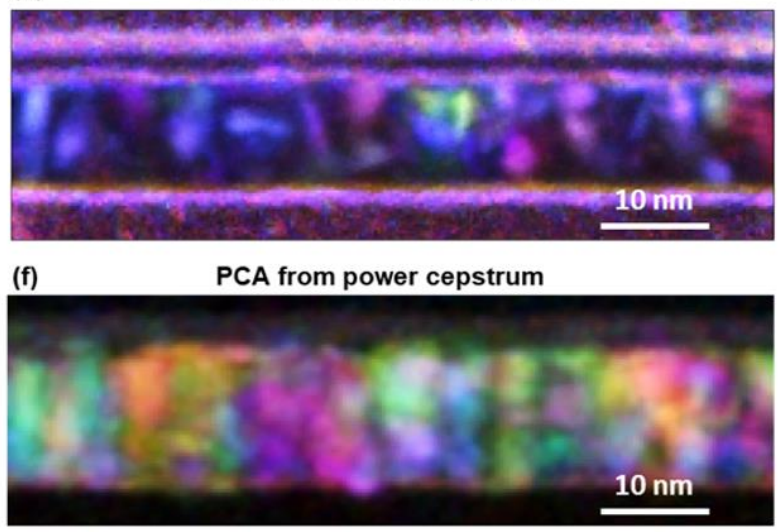

Figure 2. More robust grain mapping using EWPC transform on 4D-STEM nanodiffraction data sets. (a) A Dark-Field diffraction map from the highlighted diffraction pixel in Fig. 2b. (b) A diffraction pattern from the red spot location in Fig. 2a showing substantial sample tilt. (c) A real-space Dark Field Cepstral map reconstructed from the cepstral peak marked by the red spot in Fig. 2d, showing much common grain structure over a wider mistitle range than in Fig. 2a. (d) A power cepstrum generated from the NBED at the red spot in Fig. 2c (which is in the same position as in Fig. 2a) showing a complete pattern despite the sample mistilt. (e, f) A mapping of grains by adding up the first 30 components characterized by Principle Component Analysis (PCA) on the original diffraction data (e) and after a cepstrum transform (f). The cepstrum transform successfully detects more grain structures, being less sensitive to grain tilt and thickness variations. The columnar nature of the grains is now clearer. 


\section{References}

[1] E. Padgett et al., The Exit-Wave Power-Cepstrum Transform for Scanning Nanobeam Electron Diffraction. Part 1: Robust Strain Mapping at Subnanometer Resolution and Subpicometer Precision, arXiv:1911.00984 (2019).

[2] K.-H. Kim et al., TEM based high resolution and low-dose scanning electron nanodiffraction technique for nanostructure imaging and analysis, Micron 71(2015), 39-45.

[3] P. Cueva et al., Data Processing for Atomic Resolution Electron Energy Loss Spectroscopy, Microscopy and Microanalysis 18 (2012), 667-675.

[4] Research supported by CCMR DMR-1719875, DOE grants DE-EE0007271, and DE-SC0019445. 